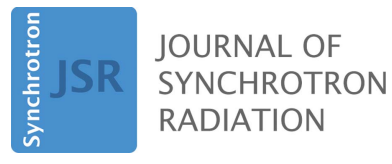

ISSN 1600-5775

Received 4 November 2020

Accepted 12 February 2021

Edited by K. Kvashnina, ESRF - The European Synchrotron, France

Keywords: XAFS; QXAFS; in situ/operando.

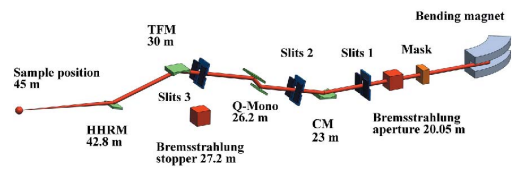

OPEN $\odot$ ACCESS

\section{The new X-ray absorption fine-structure beamline with sub-second time resolution at the Taiwan Photon Source}

\author{
Chih-Wen Pao, ${ }^{a *}$ Jeng-Lung Chen, ${ }^{a}$ Jyh-Fu Lee, ${ }^{a}$ Meng-Che Tsai, ${ }^{b}$ Chi-Yi Huang, ${ }^{a}$ \\ Chao-Chih Chiu, ${ }^{a}$ Chao-Yu Chang, ${ }^{a}$ Liang-Chih Chiang ${ }^{\mathrm{a}}$ and Yu-Shan Huang ${ }^{\mathrm{a}}$
}

\footnotetext{
aNational Synchrotron Radiation Research Center, Hsin-Ann Road, Hsinchu Science Park, Hsinchu 30076, Taiwan, and ${ }^{\mathbf{b}}$ NanoElectroChemistry Lab, Graduate Institute of Applied Science and Technology, National Taiwan University of Science and Technology, Taipei, Taiwan. *Correspondence e-mail: pao.cw@nsrrc.org.tw
}

The new TPS 44A beamline at the Taiwan Photon Source, located at the National Synchrotron Radiation Research Center, is presented. This beamline is equipped with a new quick-scanning monochromator (Q-Mono), which can provide both conventional step-by-step scans (s-scans) and on-the-fly scans (q-scans) for X-ray absorption fine-structure (XAFS) spectroscopy experiments, including X-ray absorption near-edge structure (XANES) and extended X-ray absorption fine-structure (EXAFS) spectral measurements. Ti and Te $K$-edge XAFS spectra were used to demonstrate the capability of collecting spectra at the limits of the working energy range. The $\mathrm{Ni}$ and $\mathrm{Cu} K$-edge XAFS spectra for a $\mathrm{Cu}$-doped $\mathrm{Pt} / \mathrm{Ni}$ nanocomposite were acquired to test the performance of the newly commissioned beamline. Pt $L_{3}$ - and Ru $K$-edge quick-scanning XAFS (QXAFS) spectra for standard $\mathrm{Pt}$ and $\mathrm{Ru}$ foils, respectively, revealed the stability of the q-scan technique. The results also demonstrated the beamline's ability to collect XAFS spectra on a sub-second timescale. Furthermore, a $\mathrm{Zn}_{(\mathrm{s})}\left|\mathrm{Zn}^{2+}{ }_{(\mathrm{aq})}\right| \mathrm{Cu}_{(\mathrm{s})}$ system was tested to indicate that the states of the $\mathrm{Zn}$ electrode could be observed in real time for charging and discharging conditions using an in situ/operando setup combined with QXAFS measurements.

\section{Introduction}

X-ray absorption fine-structure (XAFS) spectroscopy, which includes X-ray absorption near-edge structure (XANES) and extended X-ray absorption fine structure (EXAFS), is an important experimental method used at synchrotron radiation facilities. Over the last 40 years, this method has been widely applied to scientific research and industry applications (Lee $e t$ al., 1981). The XAFS spectral measurements require scanning of X-ray photon energies over a range of $1000 \mathrm{eV}$; these photon energies are selected using a monochromator. Conventional monochromators are driven by stepper motors, and the absorption coefficients of each energy value are measured at every step. A full XAFS spectrum can be obtained in a time ranging from a few minutes to ten minutes, depending on the experimental setup and the sample type. Recently, a quick-scanning monochromator (Q-Mono) has been successfully developed, which allowed a single spectrum to be acquired in the sub-second time scale, in contrast to the traditional acquisition time which can take tens of minutes (Müller et al., 2015; Khalid et al., 2010; Nonaka et al., 2016). Thus, this evolution of XAFS spectroscopy into 'quick-scanning XAFS (QXAFS)' provides a powerful tool for timeresolved XAFS measurements. The local environment of the absorbing atoms in a sample can be tracked in real time using 
an in situ setup combined with the QXAFS technique. This can potentially facilitate experimental measurements under in situ or operando environments for monitoring various processes such as the charge and discharge cycles of batteries and chemical processes in catalysts (Eslava et al., 2016; Marchionni et al., 2020; Gaur et al., 2019).

The theory and first experiment using QXAFS was proposed and performed by Ronald Frahm in 1988 (Frahm, 1988). In typical XAFS experiments the spectra of Co and Fe standard foils can be obtained in a few seconds. Several quickscanning beamlines have been built around the world over the last ten years to facilitate QXAFS measurements. The capability of quickly scanning the energy of X-ray photons has been achieved with different results via different designs of QMono. For example, a group at NSLS developed a cam system driven by a DC motor that was installed at the $\mathrm{X} 18 \mathrm{~B}$ beamline (Khalid et al., 2010). The spectrum can be collected both in the low to high (up spectrum) and the high to low (down spectrum) energy ranges, with each scan spanning $100 \mathrm{~ms}$. The Toyota beamline at SPring- 8 features a Q-Mono driven by high-speed AC servo motors, and it is capable of data acquisition in the sub-second range (Nonaka et al., 2016). Recently, a Q-Mono with a direct drive and goniometer has been installed in the SuperXAS (SLS) and P64 Advance XAFS (DESY) beamlines. This enables very stable measurements in both the conventional step-by-step scan (s-scan) mode and the on-the-fly scan (q-scan) mode (Müller et al., 2016; Bornmann et al., 2019).

Experimental techniques such as energy-dispersive X-ray absorption spectroscopy (EDXAS) and pump-probe X-ray absorption spectroscopy (XAS) improve time resolution from the sub-second scale to the nanosecond scale or lower (Pascarelli et al., 2016; Lima et al., 2011; Smolentsev et al., 2013). However, the EDXAS setup does not allow for measurements of the fluorescence signal in samples, and it requires a very flat sample surface. Pump-probe XAS can only be used for samples that can be excited by pulse laser, such as photocatalysts (Uemura et al., 2020; Smolentsev et al., 2018). The QXAFS technique can be more flexible and convenient for in situ/operando XAFS measurements. We have developed and constructed a QXAFS beamline (TPS 44A) using a bending-magnet source at the newly constructed Taiwan Photon Source (TPS). This beamline has been commissioned to provide a highly flexible and stable experimental facility
Table 1

Beamline specifications of TPS 44A.

\begin{tabular}{ll}
\hline Energy range & $4.5-34 \mathrm{keV}$ \\
Beam spot at focal point & $100 \mu \mathrm{m}(\mathrm{H}) \times 300 \mu \mathrm{m}(\mathrm{V})$ \\
Beam spot at sample position & $1.7 \mathrm{~mm}(\mathrm{H}) \times 1.15 \mathrm{~mm}(\mathrm{~V})$ \\
Photon flux & $3 \times 10^{11} \mathrm{photons} \mathrm{s}{ }^{-1}$ at $10 \mathrm{keV} \dagger$ \\
Q-Mono & Channel-cut Si(111) crystal \\
Energy resolution & $1.406 \times 10^{-4}$ at $5 \mathrm{keV} \ddagger$ \\
High harmonic rejection ratio & $<10^{-4}$ \\
Time to collect single spectrum & $50 / 10 \mathrm{~ms}($ EXAFS/XANES) \\
\hline
\end{tabular}

$\dagger$ Measured with $500 \mathrm{~mA}$ top-up mode in the storage ring. $\$$ The value is obtained from the result of $S H A D O W$ calculations.

that permits the acquisition of QXAFS and conventional XAFS spectra.

\section{Beamline}

The TPS 44A beamline is designed to provide an X-ray photon source with a stable and wide energy range for QXAFS measurements. The bending magnet is therefore used as a photon source for this beamline. The optical components include a bendable collimating mirror $(\mathrm{CM})$, a channel-cut Q-Mono, two bendable toroidal focusing mirrors (TFMs), and a high-harmonic-rejection mirror (HHRM). To measure the $K$ - or $L$-edge XAFS spectra of the elements in the periodic table to the maximum extent possible, we set the working energy of this beamline to range from 4.5 to $34 \mathrm{keV}$. The construction and commissioning of the beamline were completed at the end of 2018. The optical layout and the coordinate axes are displayed in Fig. 1. In addition, Table 1 shows the beamline specification.

\subsection{Photon source}

The TPS machine is currently operating at the electron energy of $3 \mathrm{GeV}$ (Horiuchi, 2015). The electron beam current is $400 \mathrm{~mA}$ in the top-up operation mode. The magnetic peak field and critical energy of the bending magnet is $1.198 \mathrm{~T}$ and $7.12 \mathrm{keV}$, respectively, and it is used as the photon source for the TPS 44A beamline. The low power density of the bending magnet is advantageous because it reduces the thermal deformation and improves the stability of the optics, with the broad spectrum requiring no complex control like undulator gap tuning. The feasible length of the TFM and its small (a)

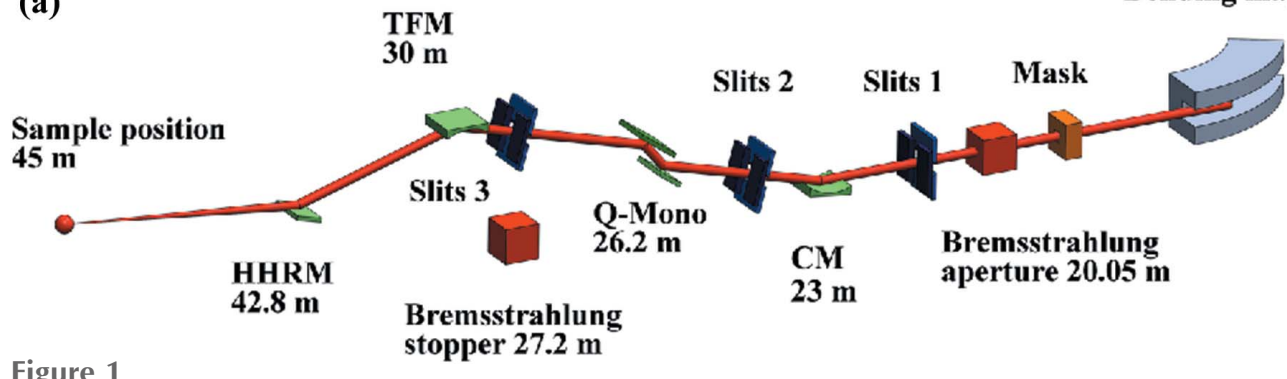

\section{Bending magnet}

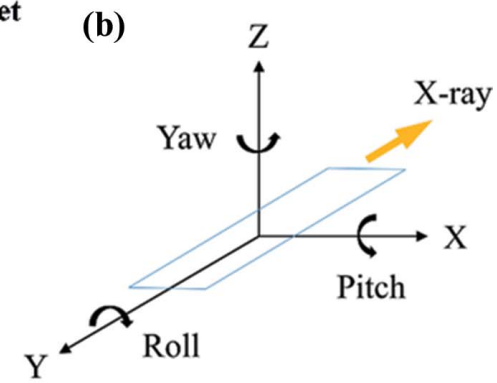

(a) Schematic of the optical design configuration, where CM is a collimating mirror, Q-Mono is a quick-scanning monochromator, and TFM is a toroidal focusing mirror. $(b)$ The coordinate axes of the system. 
sagittal curvature limit the photon acceptance of beamline, so the effective angular opening is $1 \mathrm{mrad} \times 0.11 \operatorname{mrad}(\mathrm{H} \times \mathrm{V})$.

\subsection{Collimating mirror}

The collimating mirror is the first optical device in this beamline. It is used to collimate the divergence of the bendingmagnet radiation in the vertical direction so that the energy resolution can satisfy the experimental needs. To ensure radiation safety and the operational convenience of the beamline, some essential components are placed before the $\mathrm{CM}$. Thus, the $\mathrm{CM}$ is placed $23 \mathrm{~m}$ from the source. The reflectivity of the mirror decreases with the grazing angle as well as energy. A smaller grazing angle results in higher reflectivity and a longer mirror length. Manufacturing highquality long mirrors is a challenge. By setting the CM's grazing angle to $2.5 \mathrm{mrad}$, the system can create a compromise between the feasible mirror length and the photon flux. The effective length of the $\mathrm{CM}$ is set to $100 \mathrm{~cm}$, and the mirror faces upward. The $\mathrm{CM}$ is bendable, and the meridional radius is $18.4 \mathrm{~km}$ with a $2.5 \mathrm{mrad}$ grazing angle. To minimize thermal deformations in the CM, a side water-cooling system is incorporated. To account for the high harmonic rejection, the mirror includes three reflection stripes, namely $\mathrm{Si}, \mathrm{Rh}$ and $\mathrm{Pt}$ coating on a single silicon substrate.

\subsection{Quick-scanning monochromator}

The Q-Mono is the key component of this beamline. It is located $26 \mathrm{~m}$ from the source, allowing it to select the photon energies via a channel-cut crystal with a Si (111) diffraction plane. The channel-cut crystal is driven by a torque motor mounted at the center of the goniometer, which enables the Q-Mono to perform conventional s-scans and q-scans for frequencies up to $50 \mathrm{~Hz}$ (Müller et al., 2015). The s-scan XAFS measurements are performed using a Huber goniometer driven by the stepper motor with a step/encoder resolution of $5 \times 10^{-5}$ degrees. The torque motor is driven by a sine wave signal and can scan smoothly in different angular ranges at different goniometer angles. The stage of channel-cut crystal is connected to the torque motor, to ensure rapid photon energy scanning. Although the angular scan range of the torque motor depends on the Bragg angle and the scanning speed, the maximum angular range of this motor can reach up to $4.2^{\circ}$, which can be used for energies scanning for $800 \mathrm{eV}$ at $\mathrm{Ti}$ $K$-edge spectral measurements.

\subsection{Toroid focusing mirrors}

This focusing mirror is placed $30 \mathrm{~m}$ from the source and is composed of two bendable toroid mirrors with $\mathrm{Rh}$ and $\mathrm{Pt}$ coatings, respectively. The grazing angle of the TFM is $2.5 \mathrm{mrad}$, like that of the CM. The focusing mirror has a demagnification ratio of $1: 2$ to minimize aberration in the horizontal direction. In the vertical direction, the $\mathrm{CM}$ and TFM form a focal spot $45 \mathrm{~m}$ from the source. Finally, the X-ray beam is focused to an area of $100 \mu \mathrm{m} \times 300 \mu \mathrm{m}(\mathrm{H} \times \mathrm{V})$ at the focal point of the TFM. The Q-Mono uses the channel-cut crystal to select the photon energy, so the distance between
Table 2

Relationship between photon energy range, beam offset and the variation in the beam offset in the vertical direction.

\begin{tabular}{lll}
\hline $\begin{array}{l}\text { Energy range } \\
(\mathrm{keV})\end{array}$ & $\begin{array}{l}\text { Beam offset } \\
(\mathrm{mm})\end{array}$ & $\begin{array}{l}\text { Beam offset } \\
\text { variation }(\mu \mathrm{m})\end{array}$ \\
\hline $4.5-5.5$ & $17.068-17.730$ & 649 \\
$5.5-7.5$ & $17.730-18.328$ & 598 \\
$7-11$ & $18.226-18.691$ & 465 \\
$10-34$ & $18.625-18.968$ & 343 \\
\hline
\end{tabular}

the two diffraction planes is fixed. Therefore, the beam offset of the Q-Mono varies with photon energy during the XAFS measurements. The vertical position of the TFM is therefore tailored for the change of the beam height. For operation convenience, the TFM is set at a moderate vertical position during the energy scan. Table 2 demonstrates the relationship between the photon energy range, beam offset and the variation in the beam offset. The maximum difference in the beam offset is approximately $700 \mu \mathrm{m}$ during a $1000 \mathrm{eV}$ energy scan. The sample position is placed $44 \mathrm{~m}$ from the photon source, and the beam size is $1.7 \mathrm{~mm} \times 1.15 \mathrm{~mm}(\mathrm{H} \times \mathrm{V})$. The beam profile is shown in Fig. 2. Fig. 3 displays the beam profile in the vertical direction at photon energies of 8779 and $9979 \mathrm{eV}$. These two values are the starting and ending photon energy of the $\mathrm{Cu} K$-edge XAFS spectrum. Fig. 3 shows that the
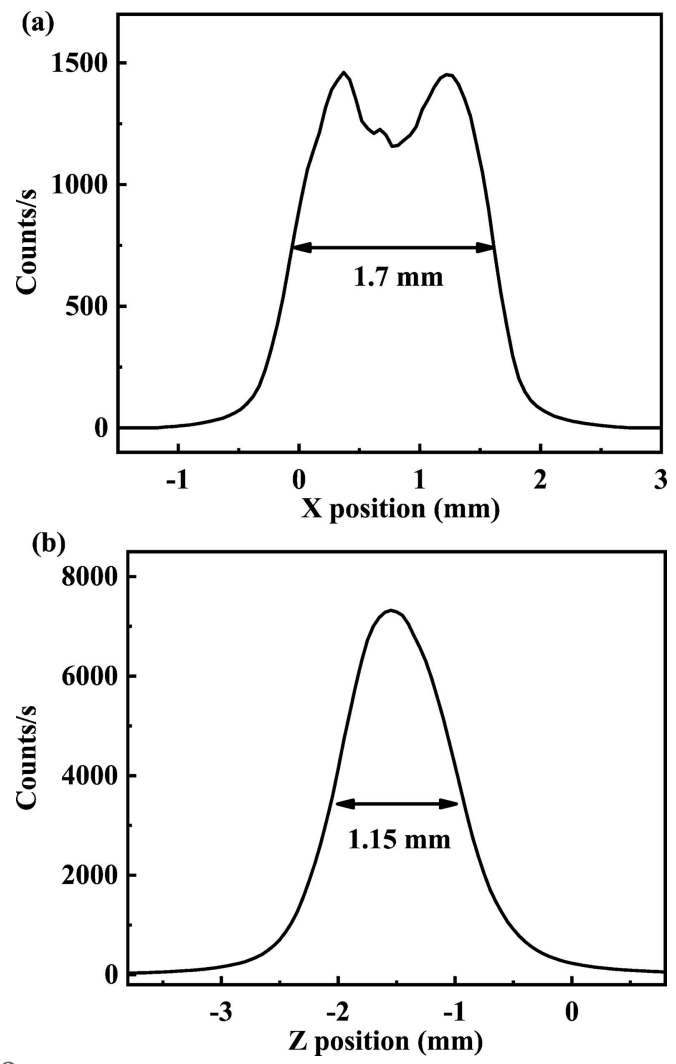

Figure 2

The X-ray beam profile of $(a)$ the horizontal direction and $(b)$ the vertical direction at the sample position. The sample position is $44 \mathrm{~m}$ from the photon source, and the focal point of the TFM is $45 \mathrm{~m}$ from the photon source. The curves were obtained at a photon energy of $8979 \mathrm{eV}$ with slit openings of $10 \mu \mathrm{m}$ and a scanning step of $10 \mu \mathrm{m}$. 


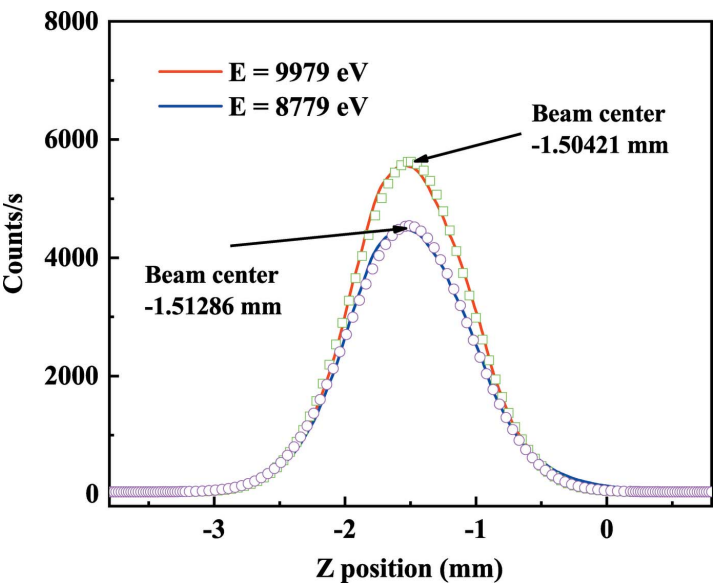

Figure 3

The vertical beam profile at the sample position. The blue and red lines are the profiles measured at a photon energy of $8779 \mathrm{eV}$ and $9979 \mathrm{eV}$, respectively. The green squares and purple circles are the best Gaussian fitting.

beam center almost coincides during spectral measurements for $\mathrm{Cu} K$-edge XAFS. The Gaussian fitting result shows that the variation in the beam offset between the photon energy of the starting and ending points of the $\mathrm{Cu} K$-edge XAFS spectrum is $10 \mu \mathrm{m}$. According to this measurement and an optical simulation, the beam position at the sample position is not altered much, and the photon loss is acceptable for this difference in beam offset variation.

\subsection{High-order harmonic rejection mirror}

The HHRM is used to reduce the contaminations of highorder photons at the working energy lower than $8 \mathrm{keV}$. Two important approaches for rejecting high-order harmonic photons include the crystal detuning of double-crystal monochromators and the use of grazing-incidence mirrors. Like for the beam offset variation issue, the crystal detuning method cannot be applied for the channel-cut crystal. In this setup, the grazing-incidence mirrors are used for high harmonics rejection (Hastings et al., 1978; Latimer et al., 1995; Lamble, 1995). For this beamline, the CM has three reflection surfaces, namely $\mathrm{Si}, \mathrm{Rh}$ and $\mathrm{Pt}$, and the coating surfaces of the two TFMs are $\mathrm{Rh}$ and $\mathrm{Pt}$, respectively. To suppress the reflection of high harmonic photons, the corresponding reflective surface on the $\mathrm{CM}$ is selected in conjunction with the specific coating of the TFM. Under such an operation, a high harmonics rejection ratio (3rd/1st) lower than $10^{-4}$ can be achieved in the working energy above $8 \mathrm{keV}$. However, at low energies, it is still difficult to reach our goal using these combinations. An extra plane mirror with $\mathrm{Rh}$ and Si stripes was therefore inserted before the sample position to further suppress high harmonics. The plane mirror has a fixed grazing angle of $4.5 \mathrm{mrad}$ and reflects upward. The harmonic ratios for different photon energies, simulated by the SHADOW (Sanchez del Rio et al., 2011) software, are shown in Fig. 4. The harmonic content is reduced to $10^{-4}$ or less for photon energies greater than $4.8 \mathrm{keV}$. Based on the results of the simulation, five operation modes, shown in Table 3 , are proposed, in
Table 3

Selection of reflective stripes according to photon energies.

\begin{tabular}{llll}
\hline $\begin{array}{l}\text { Operation energy } \\
\text { range }(\mathrm{keV})\end{array}$ & $\begin{array}{l}\mathrm{CM} \\
(2.5 \mathrm{mrad})\end{array}$ & $\begin{array}{l}\text { TFM } \\
(2.5 \mathrm{mrad})\end{array}$ & $\begin{array}{l}\text { HHRM } \\
(4.5 \mathrm{mrad})\end{array}$ \\
\hline $4.8-7$ & $\mathrm{Si}$ & $\mathrm{Rh}$ & $\mathrm{Si}$ \\
$6.2-12$ & $\mathrm{Si}$ & $\mathrm{Rh}$ & $\mathrm{Rh}$ \\
$8.2-12$ & $\mathrm{Si}$ & $\mathrm{Rh}$ & \\
$10.5-23$ & $\mathrm{Rh}$ & $\mathrm{Rh}$ & \\
$22-34$ & $\mathrm{Pt}$ & $\mathrm{Pt}$ & \\
\hline
\end{tabular}

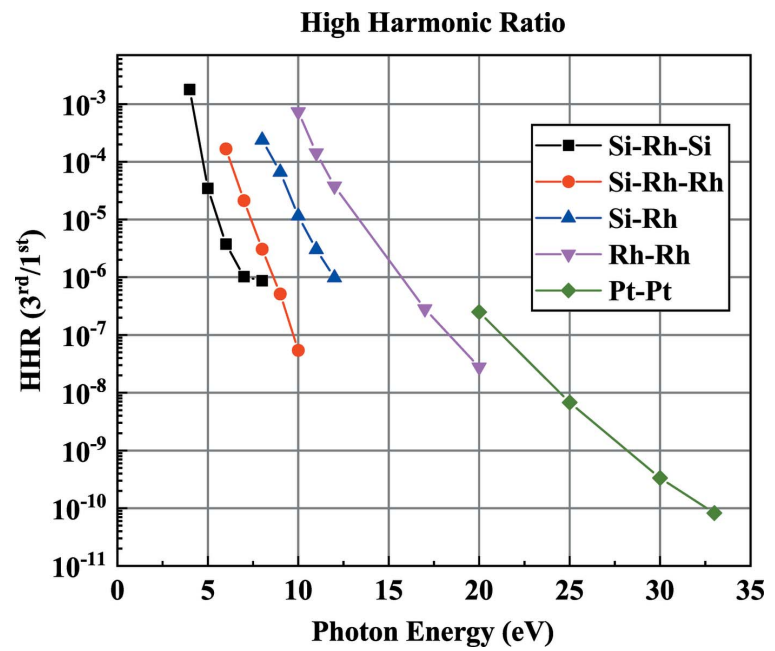

Figure 4

Harmonic rejection ratio (3rd/1st) at the sample position simulated by SHADOW with both the collimating mirror and the toroidal focusing mirrors set at a grazing angle of $2.5 \mathrm{mrad}$ and with the HHRM set at $4.5 \mathrm{mrad}$.

which the mirror stripes are switched according to the working energy.

\subsection{Beamline control}

The Experimental Physics and Industrial Control System (EPICS) was used as the control system for this beamline. Almost all beamline components, such as motors, counters and various equipment controllers, are controlled by EPICS, which provides low-level instrumentation of the beamline components by interfacing various electronic hardware components, including motor controllers, screen-monitoring CCDs, and current amplifiers. There are several high-level data collection Python scripts based on EPICS that can be used to describe experimental procedures.

\section{Experimental end-station and beamline performance}

The experimental end-station is dedicated to measuring the XAFS spectrum in the s-scan and q-scan modes. All components are placed on a motorized optical table. A set of $X-Z$ slits is located at the front of the optical table and is used to select the central portion of the mono X-ray beam. The slits can minimize the effect of the beam offset variations associated with photon energy on the data quality of the XAFS spectrum. The rise time of the gridded ionization chamber is roughly two orders of magnitude faster than comparable 
parallel plate ionization chambers (Müller et al., 2013). The gridded ionization chambers are therefore used to detect the photon flux for both the q-scan and s-scan modes. ISEG THQseries high voltage power is supplied with a two-output channel and is used to create a potential on the cathode and grid. The ionization chamber is always operated at saturation voltage. The output signal of the gridded ionization chamber is collected using a Keithley 428 current amplifier. This setup is used to perform the XAFS spectral measurements in the transmission mode. To obtain the QXAFS spectra in the fluorescence mode, a pin diode is used as the fluorescence detector. For samples with very low elemental concentrations (a few to several thousand p.p.m.), the spectra were obtained using a seven-element silicon drift detector (SDD) in the sscan mode. For the s-scans, the voltage signals from the current amplifiers are converted into frequencies using voltage-to-frequency converters (Tsuji Electronics VF8-01) and digitized using a counter (Tsuji Electronics CT08-01E). The procedure for the s-scan data collection is described by Python scripts based on EPICS. The data acquisition system (DAQ) for the q-scan mode is separate from the beam control system (Stötzel et al., 2011; Müller et al., 2016). The acquired signals are digitized using a 16-bit analog-to-digital converter board (National Instruments PXIe-6366) with a maximum sampling rate of $2 \mathrm{MS} \mathrm{s}^{-1}$ per channel. The signals for the angular encoders on the Q-Mono are recorded simulta- (a)

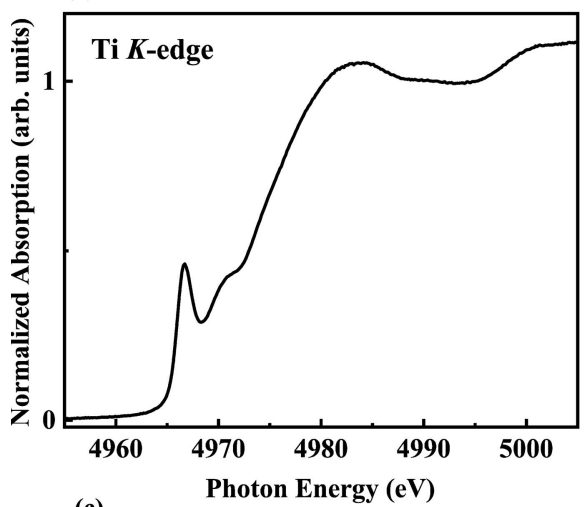

(c)

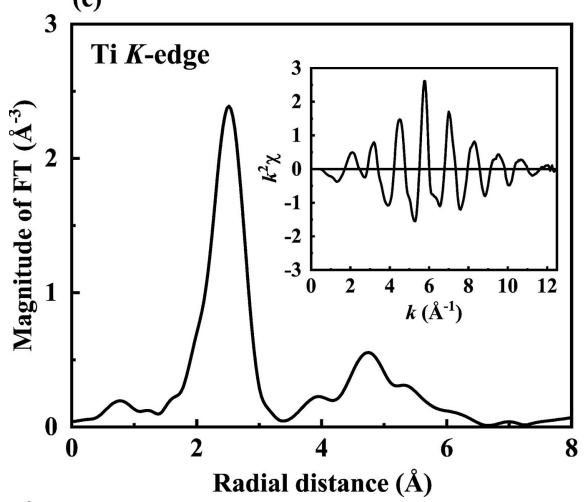

(b)

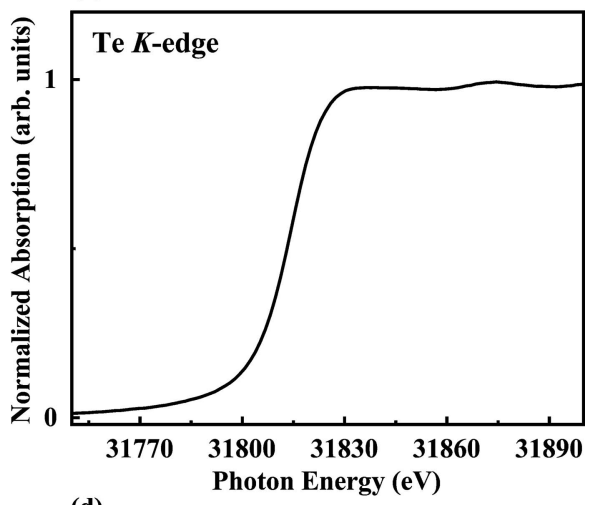

(d)

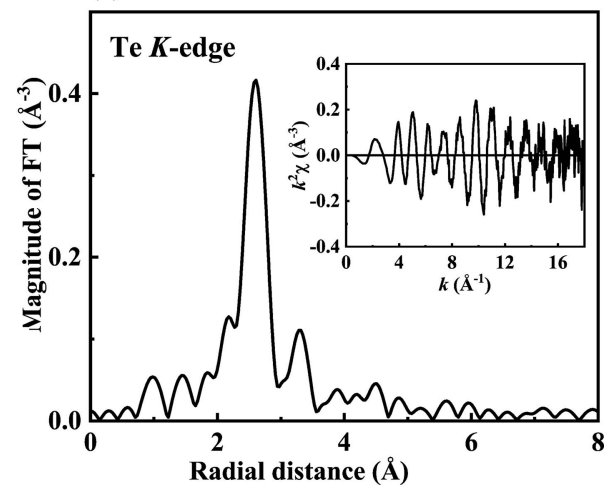

Figure 5

Normalized $\mathrm{Ti}(a)$ and Te $(b) K$-edge XANES spectra of Ti standard foil and Te powder. Panels $(c)$ and $(d)$ are the Fourier transform (FT) spectra of Ti standard foil and Te powder at the $K$-edge EXAFS spectra. The insets in $(c)$ and $(d)$ show the EXAFS $k^{2} \chi$ data. The FT range is $3-12 \AA^{-1}$ and 4-15 $\AA^{-1}$ for the Ti and Te $K$-edge EXAFS spectra, respectively. The data were averaged through 120 spectra obtained in q-scan mode at a Q-Mono oscillation frequency of $1 \mathrm{~Hz}$. neously. The NIPXIe-1073 chassis includes a $10 \mathrm{MHz}$ reference clock, which can perfectly synchronize all devices in the DAQ. Once the HHRM reduces the high-order photons contamination, the mono X-ray beam refracts upwards by 9 mrad. The height and pitch angle of the optical table can be optimized to maintain the relative position of the desktop and X-ray beam.

The working energy range of this beamline spans from 4.5 to $34 \mathrm{keV}$, which is a range suitable for researching topics related to titanium and tellurium elements. Fig. 5 presents the results of $K$-edge absorption measurements for Ti standard foil and Te powder. This figure demonstrates that the TPS 44A can collect spectra across the range from the lowest to highest photon energies. The spectra were obtained in transmission mode using q-scan at a Q-Mono oscillation frequency of $1 \mathrm{~Hz}$, and 120 spectra were averaged together. The following two cases were used to test the performance of the q-scan mode.

\subsection{Cu-doped Pt/Ni nanocomposite}

We acquired the Ni $K$-edge XAFS spectra of a Cu-doped $\mathrm{Pt} / \mathrm{Ni}$ nanocomposite to test the performance of the q-scan experimental method. The sample contained $\mathrm{Pt}, \mathrm{Cu}$ and $\mathrm{Ni}$ with elemental concentrations of $14,0.37$ and 2.71 wt\%, respectively. Fig. 6 displays the normalized Ni $K$-edge XAFS spectra obtained using the s-scan and q-scan methods in the transmission mode. The q-scan data were collected using a Q-Mono oscillating at $1 \mathrm{~Hz}$ for $1 \mathrm{~min}$. Two complete spectra were acquired per second, resulting in 120 averaged spectra with an improved signal-to-noise $(\mathrm{S} / \mathrm{N})$ ratio, as illustrated in Fig. 6. By contrast, the time to obtain one spectrum in the sscan mode was approximately $30 \mathrm{~min}$ compared with 1 min required by the qscan mode. The resultant spectra of the two modes perfectly overlapped in both the XANES and EXAFS regions, indicating the reliability and reproducibility of the q-scan method. Therefore, we successfully developed a q-scan technique that resulted in a significant decrease in the data collection time, improving the operating efficiency of the beamline. The rapid data acquisition rate can also reduce radiation-induced damage in biological research; thus, this technology facilitates investigations in new areas of research. The right-hand inset of Fig. 6 shows the Ni $K$-edge $k^{3}$-weighted EXAFS spectrum of a $\mathrm{Cu}$-doped $\mathrm{Pt} / \mathrm{Ni}$ nanocomposite. The spectra collected using the q-scan method are considerably smoother than those collected using s-scan, particularly in the region in which the $k$-value is 


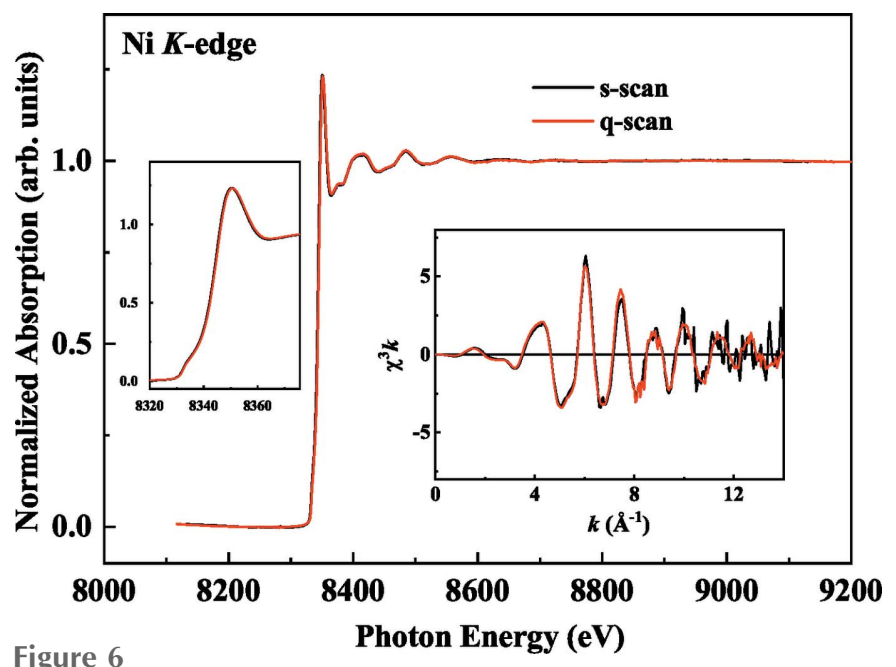

Normalized Ni $K$-edge XAFS spectrum of a Cu-doped Pt/Ni nanocomposite obtained using the traditional s-scan (black) and the q-scan (red) methods. The left inset shows a zoomed-in image of the XANES range, and the right inset presents the $k^{3}$-weighted EXAFS spectra. The q-scan data were obtained by averaging 120 spectra at a Q-Mono oscillation frequency of $1 \mathrm{~Hz}$.

greater than 8 , indicating that the q-scan experimental method can generate better quality data in addition to increasing the rapidity of the data acquisition.

Conventional methods such as transmission and fluorescence XAFS are used to measure samples having high and low elemental concentrations, respectively (Bunker, 2010). In general, the transmission mode is not suitable for investigating a sample with a white line edge-jump smaller than 0.1. Fig. 7(a) presents the $\mathrm{Cu} K$-edge XANES spectrum of the $\mathrm{Cu}$-doped $\mathrm{Pt} /$ $\mathrm{Ni}$ nanocomposite after $1 \mathrm{~min}$ in the transmission mode using the q-scan acquisition method. Although the white line edgejump of the absorption coefficient was only 0.03 , the XANES region showed a highly smooth spectrum. The EXAFS oscillations exhibit a clear phase and strong amplitude, as illustrated in Fig. 7(b), indicating that the data are suitable for EXAFS processing. XAFS measurements in samples with elemental concentrations less than $1 \mathrm{wt} \%$ should be performed in the fluorescence mode using an SDD or a Lytle detector. However, because of the high intensity of the Ni $K_{\alpha^{-}}$ emission line in this nanocomposite, the SDD detector will become saturated. By contrast, because there is background from the Ni $K_{\alpha}$-emission line, the $\mathrm{S} / \mathrm{N}$ ratio of the spectrum collected using the Lytle detector is expected to be very poor. Therefore, the transmission mode using the q-scan method can be used to directly obtain the data, resulting in more rapid and effective measurements. This study demonstrates that the qscan experimental method can improve the detection limit of XAS transmission measurements.

\subsection{Time-resolved XAFS}

The QXAFS experimental method was developed mainly to provide a time-resolved technique for studying chemical processes on the sub-second timescale. Studies of chemical reactions, such as the oxidation/reduction process and ion
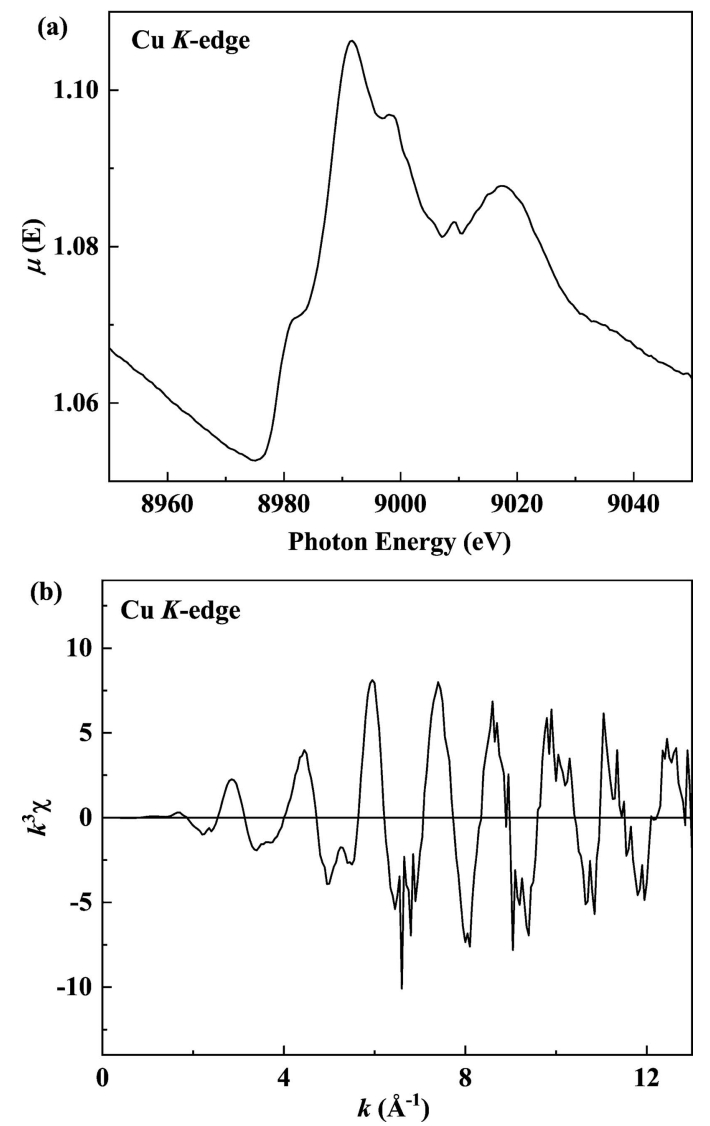

Figure 7

The $\mathrm{Cu} K$-edge $(a)$ XANES and $(b) k^{3}$-weighted EXAFS spectra of the $\mathrm{Cu}$-doped $\mathrm{Pt} / \mathrm{Ni}$ nanocomposites.

exchange/absorption, are important to researchers in the pure and applied sciences. As shown in Fig. 8, the Pt $L_{3}$-edge and $\mathrm{Ru} K$-edge XAFS spectra of standard $\mathrm{Pt}$ and $\mathrm{Ru}$ foils, respectively, were acquired to demonstrate the stability of the proposed q-scan experimental method. The up/down spectra collected at frequencies of 1 and $10 \mathrm{~Hz}$ exhibit excellent reproducibility in both the XANES and EXAFS regions, demonstrating that the TPS 44A beamline now allows users to obtain data with a sub-second time resolution. In the XANES regime, a single spectrum can be collected in $10 \mathrm{~ms}$ due to the shorter scanning range $(\sim 200 \mathrm{eV})$ compared with the EXAFS regime.

An in situ test measurement of a $\mathrm{Zn}_{(\mathrm{s})}\left|\mathrm{Zn}^{2+}{ }_{(\mathrm{aq})}\right| \mathrm{Cu}_{(\mathrm{s})}$ system during $\mathrm{Zn}$ plating/stripping on $\mathrm{Cu}$ was performed at a $1 \mathrm{~Hz}$ oscillation frequency, with the experimental setup and results shown in Fig. 9 (Wang et al., 2018; Shin et al., 2016). The $\mathrm{Cu}$ $K$-edge and $\mathrm{Zn} K$-edge XAS spectra were measured using a single scan with a photon energy range of $2000 \mathrm{eV}$ by optimizing the oscillation range of the Q-Mono. While clear features corresponding to $\mathrm{Cu}$ metal can be observed in the $\mathrm{Cu}$ $K$-edge XANES spectra [Fig. $9(a)$ ], the Zn $K$-edge XANES spectra [Fig. $9(d)$ ] show an increase in the unoccupied $4 p$ states of the $\mathrm{Zn}$ atoms during $\mathrm{Zn}$ plating/stripping. Figure $9(c)$ shows the peak intensity at a photon energy of $9667.5 \mathrm{eV}$ in Zn $K$-edge XANES spectra after standard data processing through the Demeter software package (Ravel \& Newville, 


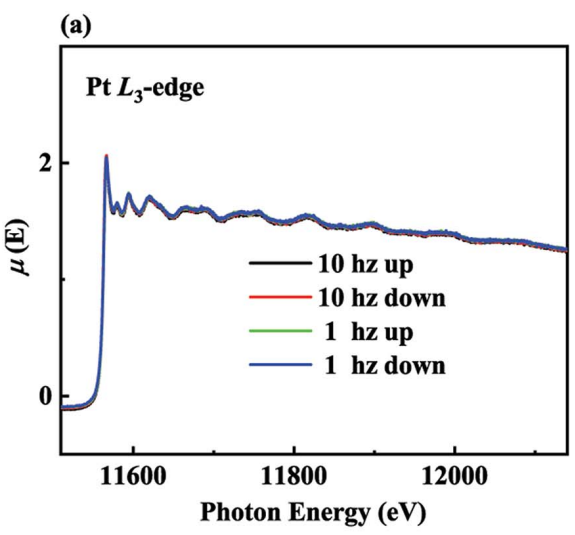

(c)

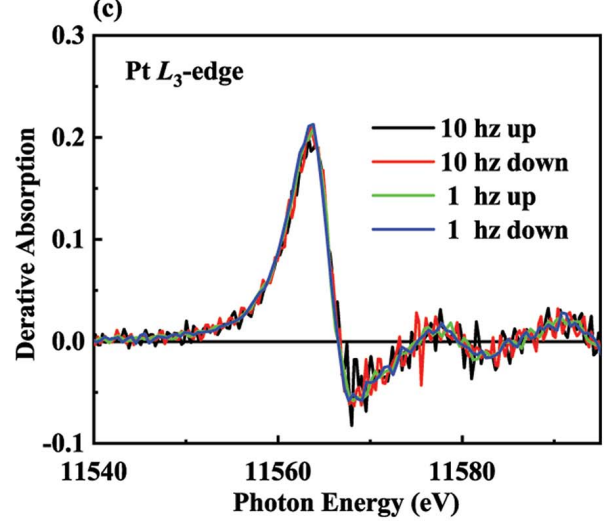

(e)

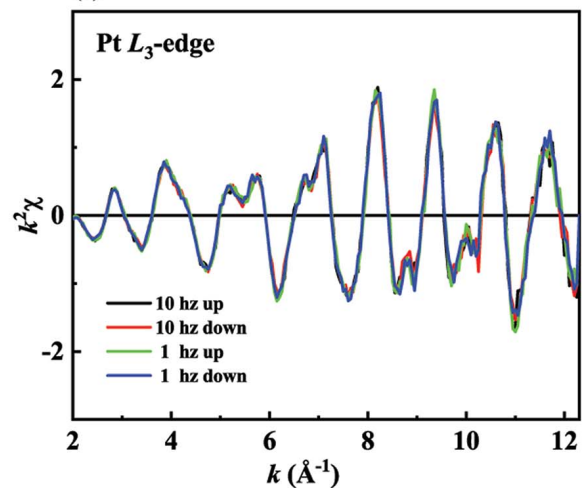

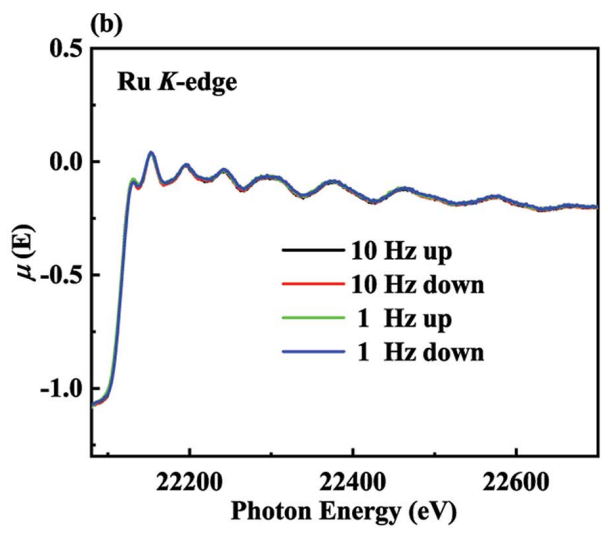

(d)
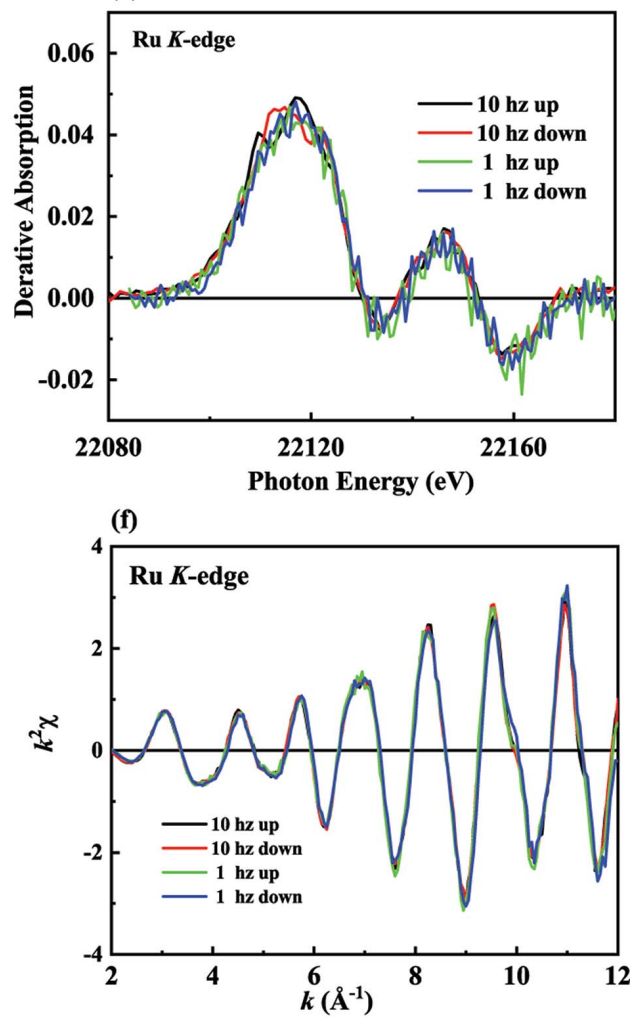

Figure 8

Single XAFS spectra of $(a) \mathrm{Pt}$ and $(b) \mathrm{Ru}$ standard foil at the Pt $L_{3}$-edge and Ru $K$-edge at Q-Mono oscillation frequencies of 1 and $10 \mathrm{~Hz}$, respectively. The up spectrum (black line) represents the sweep of the photon energy from low to high values, while the down spectrum (red line) shows the reverse trend. The normalized derivative of the XANES spectra for the $(c) \mathrm{Pt} L_{3}$-edge and $(d) \mathrm{Ru} K$-edge. The $k^{2}$-weighted EXAFS data for the $(e) \mathrm{Pt} L_{3}$-edge and $(f) \mathrm{Ru} K$-edge.

2005). The monotonically increasing intensity contains three different slopes (regions I, II and III), indicating the existence of three different $\mathrm{Zn}$ atom states at the $\mathrm{Cu}$ electrode. The plating and stripping of $\mathrm{Zn}$ on the $\mathrm{Cu}$ foil were carried out at a constant density of $1 \mathrm{~mA} \mathrm{~cm}^{-2}$ (plating step duration: $25 \mathrm{~min}$; stripping step duration: $15 \mathrm{~min}$ ). The first region corresponds to $\mathrm{Zn}_{(\mathrm{aq})}^{2+}$ in the electrolyte, the second region includes both $\mathrm{Zn}_{(\mathrm{aq})}^{2+}$ and deposited $\mathrm{Zn}$ in the plating step, while the third corresponds to the dissolution of the deposited $\mathrm{Zn}$ during the stripping step. We observed a Coulombic efficiency less than $100 \%$, indicating that $\mathrm{Zn}$ was not completely stripped from the $\mathrm{Cu}$ surface. Therefore, the spectra were not recovered to their original state. This test case showed that the states of the $\mathrm{Zn}$ electrode could be observed in real time under charging and discharging conditions via an in situ/in operando setup combined with QXAFS measurements. The experimental results provided critical information about the reaction dynamics within battery devices, which cannot be obtained through conventional XAFS measurement methods.

\section{Summary}

The TPS 44A beamline now has the ability to facilitate conventional s-scan and q-scan experimental methods for XAFS studies. The q-scan technique not only enables fast data acquisition but also improves the data quality. The combina- 


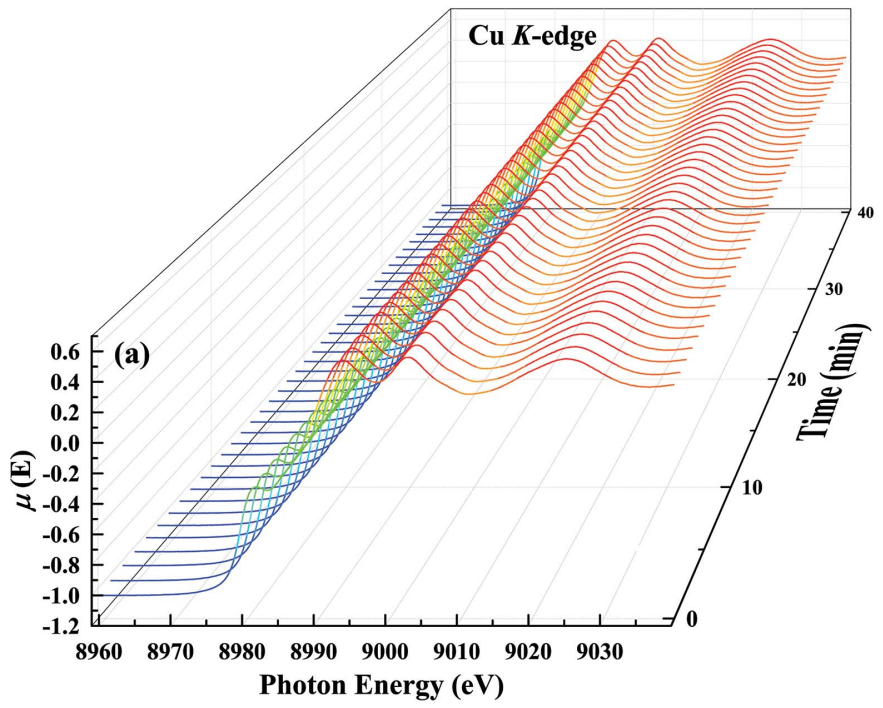

(b)
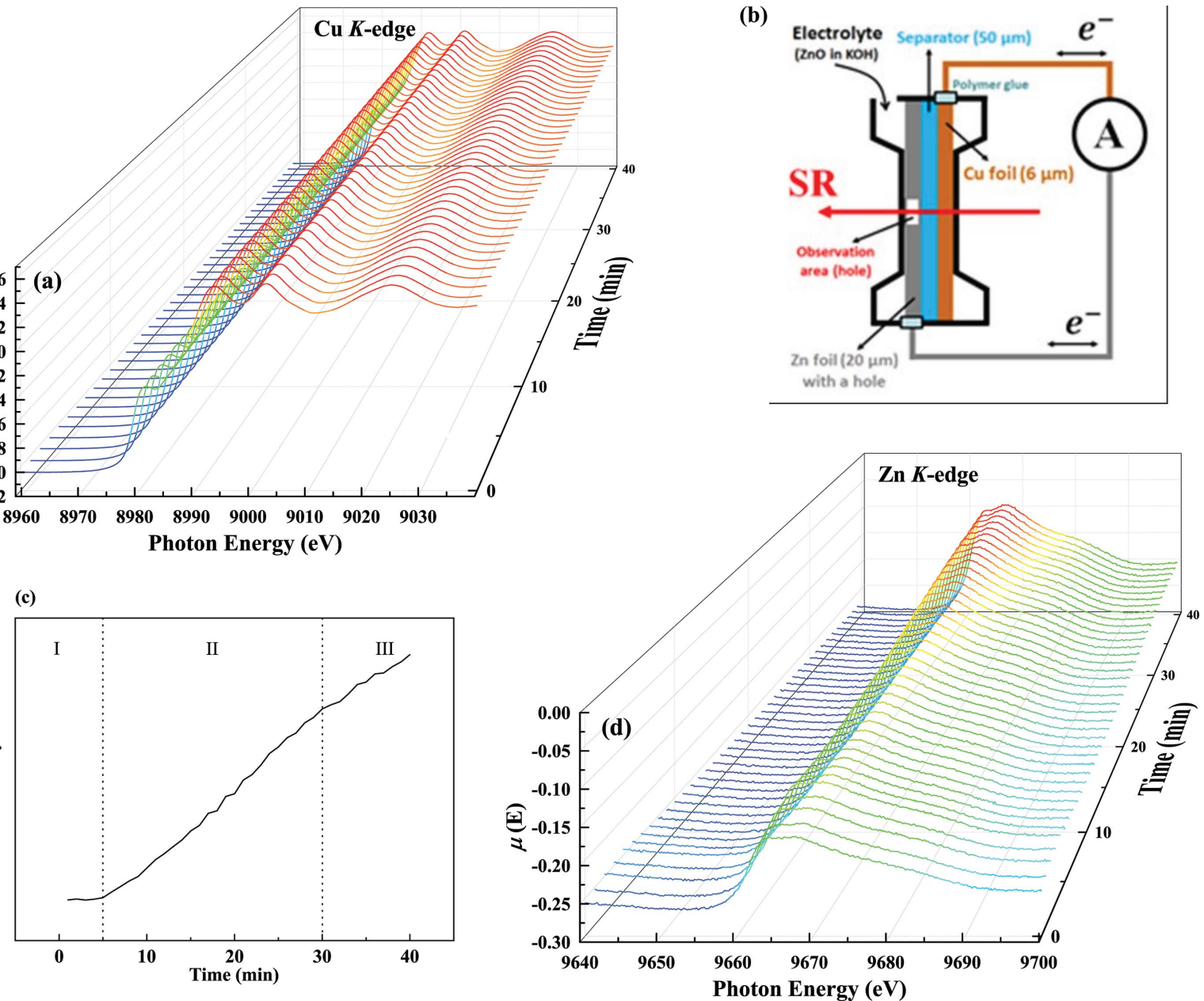

Figure 9

The in situ measurements were performed at a Q-Mono oscillation frequency of $1 \mathrm{~Hz}$. (a) The $\mathrm{Cu} K$-edge time-dependent XANES spectra of the $\mathrm{Zn}_{(\mathrm{s})}\left|\mathrm{Zn}^{2+}{ }_{(\mathrm{aq})}\right| \mathrm{Cu}_{(\mathrm{s})}$ system. (b) Schematic of the in situ measurement setup. (c) Time dependence of the $\mathrm{Zn} K$-edge XANES region after subtracting the background from the $\mathrm{Cu}$ atom signal. (d) The $\mathrm{Zn} K$-edge time-dependent XANES spectra of the $\mathrm{Zn}_{(\mathrm{s})}\left|\mathrm{Zn}^{2+}{ }_{(\mathrm{aq})}\right| \mathrm{Cu}_{(\mathrm{s})}$ system.

tion of an in situ/in operando setup and the QXAFS measurements provides a new powerful tool for time-resolved studies, which is useful for energy materials research and for observing chemical reactions on the sub-second timescale. This QXAFS technique can provide new scientific research opportunities to XAS users. Additionally, the beamline can serve as an important facility for both basic science and industrial/applied research studies.

\section{Acknowledgements}

We thank Dr Ronald Frahm and Dr Oliver Müller for their assistance with the beamline commissioning.

\section{Funding information}

We would like to thank the Ministry of Science and Technology (MoST) of Taiwan for providing financial support for the beamline construction.

\section{References}

Bornmann, B., Kläs, J., Müller, O., Lützenkirchen-Hecht, D. \& Frahm, R. (2019). AIP Conf. Proc. 2054, 040008.
Bunker, G. (2010). Introduction to XAFS: A Practical Guide to X-ray Absorption Fine Structure Spectroscopy. Cambridge University Press.

Eslava, J. L., Iglesias-Juez, A., Agostini, G., Fernández-García, M., Guerrero-Ruiz, A. \& Rodríguez-Ramos, I. (2016). ACS Catal. 6, $1437-1445$.

Frahm, R. (1988). Nucl. Instrum. Methods Phys. Res. A, 270, 578581.

Gaur, A., Hartmann Dabros, T. M., Høj, M., Boubnov, A., Prüssmann, T., Jelic, J., Studt, F., Jensen, A. D. \& Grunwaldt, J. (2019). ACS Catal. 9, 2568-2579.

Hastings, J. B., Kincaid, B. M. \& Eisenberger, P. (1978). Nucl. Instrum. Methods, 152, 167-171.

Horiuchi, N. (2015). Nat. Photon. 9, 292-293.

Khalid, S., Caliebe, W., Siddons, P., So, I., Clay, B., Lenhard, T., Hanson, J., Wang, Q., Frenkel, A. I., Marinkovic, N., Hould, N., Ginder-Vogel, M., Landrot, G. L., Sparks, D. L. \& Ganjoo, A. (2010). Rev. Sci. Instrum. 81, 015105.

Lamble, G. M. (1995). Rev. Sci. Instrum. 66, 1422-1424.

Latimer, M. J., Rompel, A., Underwood, J. H., Yachandra, V. K. \& Klein, M. P. (1995). Rev. Sci. Instrum. 66, 1843-1845.

Lee, P. A., Citrin, P. H., Eisenberger, P. \& Kincaid, B. M. (1981). Rev. Mod. Phys. 53, 769-806.

Lima, F. A., Milne, C. J., Amarasinghe, D. C., Rittmann-Frank, M. H., van der Veen, R. M., Reinhard, M., Pham, V. T., Karlsson, S., Johnson, S. L., Grolimund, D., Borca, C., Huthwelker, T., Janousch, 
M., van Mourik, F., Abela, R. \& Chergui, M. (2011). Rev. Sci. Instrum. 82, 063111.

Marchionni, V., Nachtegaal, M. \& Ferri, D. (2020). ACS Catal. 10, 4791-4804.

Müller, O., Lützenkirchen-Hecht, D. \& Frahm, R. (2015). Rev. Sci. Instrum. 86, 093905.

Müller, O., Nachtegaal, M., Just, J., Lützenkirchen-Hecht, D. \& Frahm, R. (2016). J. Synchrotron Rad. 23, 260-266.

Müller, O., Stötzel, J., Lützenkirchen-Hecht, D. \& Frahm, R. (2013). J. Phys. Conf. Ser. 425, 092010.

Nonaka, T., Dohmae, K., Hayashi, Y., Araki, T., Yamaguchi, S., Nagai, Y., Hirose, Y., Tanaka, T., Kitamura, H., Uruga, T., Yamazaki, H., Yumoto, H., Ohashi, H. \& Goto, S. (2016). AIP Conf. Proc. 1741, 030043.

Pascarelli, S., Mathon, O., Mairs, T., Kantor, I., Agostini, G., Strohm, C., Pasternak, S., Perrin, F., Berruyer, G., Chappelet, P., Clavel, C. \& Dominguez, M. C. (2016). J. Synchrotron Rad. 23, 353-368.
Ravel, B. \& Newville, M. (2005). J. Synchrotron Rad. 12, 537-541.

Sanchez del Rio, M., Canestrari, N., Jiang, F. \& Cerrina, F. (2011). J. Synchrotron Rad. 18, 708-716.

Shin, J., You, J. M., Lee, J. Z., Kumar, R., Yin, L., Wang, J. \& Shirley Meng, Y. (2016). Phys. Chem. Chem. Phys. 18, 26376-26382.

Smolentsev, G., Guda, A., Zhang, X., Haldrup, K., Andreiadis, E. S., Chavarot-Kerlidou, M., Canton, S. E., Nachtegaal, M., Artero, V. \& Sundstrom, V. (2013). J. Phys. Chem. C, 117, 17367-17375.

Smolentsev, G., van Vliet, K. M., Azzaroli, N., van Bokhoven, J. A., Brouwer, A. M., de Bruin, B., Nachtegaal, M. \& Tromp, M. (2018). Photochem. Photobiol. Sci. 17, 896-902.

Stötzel, J., Lützenkirchen-Hecht, D. \& Frahm, R. (2011). J. Synchrotron Rad. 18, 165-175.

Uemura, Y., Yokoyama, T., Katayama, T., Nozawa, S. \& Asakura, K. (2020). Appl. Sci. 10, 7818.

Wang, F., Borodin, O., Gao, T., Fan, X., Sun, W., Han, F., Faraone, A., Dura, J. A., Xu, K. \& Wang, C. (2018). Nat. Mater. 17, 543-549. 